\title{
MODEL-BASED MR IMAGE RECONSTRUCTION WITH COMPENSATION FOR THROUGH-PLANE FIELD INHOMOGENEITY
}

\author{
Jeffrey A. Fessler \\ EECS Dept., The University of Michigan \\ fessler@umich.edu
}

\author{
Douglas C. Noll* \\ BME Dept., The University of Michigan \\ dnol1@umich.edu
}

\begin{abstract}
To improve image quality in susceptibility-weighted MR imaging, it is important to correct for the effects of field inhomogeneity. In particular, susceptibility differences between air and tissue induce magnetic field nonuniformity; often those susceptibility effects have nonzero through-plane gradients that lead to spin dephasing across the slice within each voxel. If uncorrected, these through-plane gradients cause signal loss in the reconstructed images. Several methods exist for reconstructing MR images with compensation for field inhomogeneity, but most of these methods, even the model-based iterative ones, treat the inhomogeneity within each voxel as being a constant, thus ignoring the through-plane gradient effects. This paper describes a model-based iterative method for reconstructing MR images with compensation for field inhomogeneity that accounts for the slice profile and the throughplane gradients of the field inhomogeneity (assumed to be determined by a pre-scan). In particular, this paper describes an accelerated algorithm for implementing the forward model and its adjoint as needed in a conjugate gradient algorithm for iterative MR image reconstruction.
\end{abstract}

Keywords: MRI, field inhomogeneity, susceptibility, through-plane gradients, iterative image reconstruction.

\section{INTRODUCTION}

For functional magnetic resonance imaging (fMRI) based on the BOLD effect, one must use relatively late echo times to ensure good BOLD contrast. And the desire for relatively rapid dynamic imaging to track functional changes necessitates using relatively long readouts, such as spiral scans and echo-planar imaging (EPI). These characteristics of dynamic susceptibility-weighted MR imaging lead to sensitivity of the approach to magnetic field inhomogeneities. To provide good image quality in fMRI, it is important to correct for the effects of field inhomogeneity. In particular, susceptibility differences between air and tissue induce magnetic field nonuniformity; often those susceptibility effects have nonzero throughplane gradients that lead to spin dephasing across the slice

\footnotetext{
* Supported in part by NIH grants EB002683 and DA15410.
}

within each voxel. (There also can be within-plane gradients that have similar effects. For simplicity, we focus on the through-plane effects in this paper.) If uncorrected, these through-plane gradients cause signal loss in the reconstructed images. Several methods exist for reconstructing MR images with compensation for field inhomogeneity, including modelbased iterative methods, e.g., $[1,2]$ and non-iterative methods, e.g., $[3,4]$. Most of the existing methods treat the inhomogeneity within each voxel as being a constant, thus ignoring the through-plane gradient effects. An exception is previous work of Sutton et al. [5,6]; however, the algorithms described therein were relatively slow.

This paper describes a model-based iterative method for reconstructing MR images with compensation for field inhomogeneity that accounts for the slice profile and the throughplane gradients of the field inhomogeneity (assumed to be determined by a pre-scan). In particular, this paper describes an accelerated algorithm for implementing the forward model and its adjoint as needed in a conjugate gradient algorithm for iterative MR image reconstruction.

\section{MODELS}

For slice-selective MR imaging a reasonable model for the received signal is

$$
\begin{aligned}
s(t)= & \iiint h\left(z-z_{0}\right) f(x, y, z) \mathrm{e}^{-\imath \omega(x, y, z) t} \\
& \cdot \mathrm{e}^{-\imath 2 \pi\left(k_{\mathrm{X}}(t) x+k_{\mathrm{Y}}(t) y\right)} \mathrm{d} x \mathrm{~d} y \mathrm{~d} z
\end{aligned}
$$

where $h(z)$ denotes the (known) slice-selection profile, $z_{0}$ denotes the axial center of the slice, $f(x, y, z)$ denotes the (unknown) transverse magnetization, $\omega(x, y, z)$ denotes the offresonance frequency map (field map) and $\left(k_{\mathrm{X}}(t), k_{\mathrm{Y}}(t)\right)$ denotes the k-space trajectory of the scan. We assume hereafter that the field map $\omega(x, y, z)$ is known over the slice(s) of interest, based on a pre-scan of the subject, e.g., [7].

An MR scan consists of noisy samples of the above signal:

$$
y_{i}=s\left(t_{i}\right)+\varepsilon_{i}, \quad i=1, \ldots, n_{\mathrm{d}},
$$

where $\varepsilon_{i}$ denotes complex white gaussian noise and $n_{\mathrm{d}}$ denotes the number of $k$-space samples. 
Broadly speaking, the goal of MR image reconstruction is to estimate $f(x, y, z)$ from the measurement vector $\boldsymbol{y}=$ $\left(y_{1}, \ldots, y_{n_{\mathrm{d}}}\right)$. This is an ill-posed problem because the given measurements $\boldsymbol{y}$ are discrete whereas the object magnetization $f(x, y, z)$ is an unknown continuous-space function. To proceed, we parameterize the object $f(x, y, z)$ to facilitate estimation. When reconstructing a slice centered at axial position $z_{0}$, a natural series expansion is

$$
f(x, y, z)=\sum_{j=1}^{N} f_{j} b\left(x-x_{j}, y-y_{j}\right), \text { for } z \approx z_{0},
$$

where $b(\cdot)$ denotes the object basis function, $\left(x_{j}, y_{j}\right)$ denotes the center of the $j$ th basis function translate, and $N$ is the number of parameters. For simplicity, hereafter we use rect basis functions

$$
b(x, y)=\operatorname{rect}_{2}\left(\frac{x}{\Delta}, \frac{y}{\Delta}\right)
$$

(square pixels of dimension $\Delta$ ), so $N$ is the number of pixels, e.g., $128 \times 128$.

The model (3) treats the object magnetization as a constant across the slice, which will lead to some partial volume effects but seems largely unavoidable. Substituting (3) into the signal equation model (1) and simplifying leads to the model

$$
\begin{aligned}
s(t)= & \sum_{j=1}^{N} f_{j} \iint\left[\int h\left(z-z_{0}\right) \mathrm{e}^{-\imath \omega(x, y, z) t} \mathrm{~d} z\right] \\
& \cdot b\left(x-x_{j}, y-y_{j}\right) \mathrm{e}^{-\imath 2 \pi\left(k_{\mathrm{X}}(t) x+k_{\mathrm{Y}}(t) y\right)} \mathrm{d} x \mathrm{~d} y .
\end{aligned}
$$

To simplify this expression, we focus on the following series expansion for the field map:

$\omega(x, y, z)=\sum_{j=1}^{N} \operatorname{rect}_{2}\left(\frac{x-x_{j}}{\Delta}, \frac{y-y_{j}}{\Delta}\right)\left(\omega_{j}+2 \pi g_{j}\left(z-z_{0}\right)\right)$

where $\left(x_{j}, y_{j}\right)$ denotes the in-plane center of the $j$ th voxel, $\omega_{j}$ denotes the off-resonance frequency at the central slice of the $j$ th voxel, in units of $\mathrm{rad} / \mathrm{s}$, and $g_{j}$ denotes the field map through-plane gradient within the $j$ th voxel, in units of $\mathrm{Hz}$ per $\mathrm{cm}$. We can determine $\left\{\omega_{j}\right\}$ and $\left\{g_{j}\right\}$ using field map estimation methods and central differences for example [7].

Substituting (4) into the preceding signal model and simplifying leads to the signal model

$$
\begin{aligned}
s(t)= & \iint \sum_{j=1}^{N} H\left(t g_{j}\right) f_{j} \operatorname{rect}_{2}\left(\frac{x-x_{j}}{\Delta}, \frac{y-y_{j}}{\Delta}\right) \\
& \cdot \mathrm{e}^{-\imath \omega_{j} t} \mathrm{e}^{-\imath 2 \pi\left(k_{x}(t) x+k_{y}(t) y\right)} \mathrm{d} x \mathrm{~d} y \\
= & \operatorname{sinc}_{2}\left(k_{x}(t) \Delta, k_{y}(t) \Delta\right) \cdot \\
& \sum_{j=1}^{N} H\left(t g_{j}\right) \mathrm{e}^{-\imath \omega_{j} t} \mathrm{e}^{-\imath 2 \pi\left(k_{x}(t) x_{j}+k_{y}(t) y_{j}\right)} f_{j},
\end{aligned}
$$

where $H$ denotes the 1D Fourier transform of $h$.

In the absence of through-plane field gradients, i.e., if $g_{j}=0$, then the above model is equivalent to the approach described in $[1,2]$ and is thus amenable to the fast iterative algorithms described therein. However, the presence of the term $H\left(t g_{j}\right)$ prohibits direct use of those previous fast methods. The next section describes new fast methods for image reconstruction based on (5).

\section{ALGORITHMS}

\subsection{Taylor expansion}

One way to make (5) look more like a discrete Fourier transform of $f_{j}$, and thus more amenable to fast computation, is to consider a 2nd-order Taylor expansion of $H$ around the point $g_{j}=0$ :

$$
H(\nu) \approx H(0)+\frac{1}{2} \ddot{H}(0) \nu^{2}=1+\frac{1}{2} \ddot{H}(0) \nu^{2},
$$

where we assume that $\dot{H}(0)=0$ because the slice profile $h(z)$ is symmetric, and furthermore that the slice profile is normalized such that $H(0)=1$. For example, for a gaussian slice profile with $h(z)=\left(1 / \Delta_{\mathrm{Z}}\right) \mathrm{e}^{-\pi\left(z / \Delta_{\mathrm{Z}}\right)^{2}}$, we have $H(\nu)=\mathrm{e}^{-\pi\left(\Delta_{\mathrm{z}} \nu\right)^{2}}$ so $\ddot{H}(0)=-2 \pi \Delta_{\mathrm{z}}^{2}$. As another example, if $h(z)=\left(1 / \Delta_{\mathrm{z}}\right) \operatorname{rect}\left(z / \Delta_{\mathrm{z}}\right)$ then $H(\nu)=\operatorname{sinc}\left(\Delta_{\mathrm{z}} \nu\right)$ so $\ddot{H}(0)=-\Delta_{\mathrm{z}}^{2} \pi^{2} / 3$. Physically, these negative signs in the expansion reflect the signal loss associated with nonzero through-plane gradients.

Substituting (6) into (5) leads to the approximate signal model

$$
\begin{gathered}
s(t) \approx \operatorname{sinc}_{2}\left(k_{x}(t) \Delta, k_{y}(t) \Delta\right) \cdot \\
\sum_{j=1}^{N} \mathrm{e}^{-\imath \omega_{j} t} \mathrm{e}^{-\imath 2 \pi\left(k_{x}(t) x_{j}+k_{y}(t) y_{j}\right)} f_{j} \\
+\frac{1}{2} \ddot{H}(0) t^{2} \operatorname{sinc}_{2}\left(k_{x}(t) \Delta, k_{y}(t) \Delta\right) \cdot \\
\sum_{j=1}^{N} \mathrm{e}^{-\imath \omega_{j} t} \mathrm{e}^{-\imath 2 \pi\left(k_{x}(t) x_{j}+k_{y}(t) y_{j}\right)}\left(g_{j}^{2} f_{j}\right)(7)
\end{gathered}
$$

This model is now amenable to the types of fast algorithms described in $[1,2]$, because each of the summations has the same form as in the usual model-based image reconstruction with field inhomogeneity correction. Essentially, to compute the signal samples $s\left(t_{i}\right)$ using the above model requires two time-segmented NUFFT calls [1], one applied to $\left\{f_{j}\right\}$ itself, and the other applied to $\left\{g_{j}^{2} f_{j}\right\}$, along with some other simple multiplication operations.

To increase accuracy, one could use a 4th-order Taylor series, which would then require three time-segmented NUFFT calls for each signal evaluation (or adjoint thereof). Indeed, higher-order approximations may be required because for large 
through-plane field gradients, one can observe complete signal cancellation, corresponding to the zeros of the Fourier transform $H(\cdot)$ of the slice profile. For such levels of field gradients the 2nd-order approximation may be insufficient. On the other hand, tailored RF pulse design methods aim to precompensate for the dephasing (at the echo time) caused by through-plane gradients [8], so a combination of tailored RF pulses and a 2nd-order Taylor expansion might suffice.

\subsection{Basis expansion}

An alternative to the Taylor expansion approach above is to generalize any of the basis expansion approximations described in [2]. Specifically, we want to find $b_{i l}$ and $c_{l j}$ values for which

$$
H\left(t_{i} g_{j}\right) \mathrm{e}^{-\imath \omega_{j} t_{i}} \approx \sum_{l=1}^{L} b_{i l} c_{l j} .
$$

Substituting this type of approximation into (5) leads to the approximate signal model:

$$
\begin{aligned}
s\left(t_{i}\right) \approx & \sum_{l=1}^{L} b_{i l} \operatorname{sinc}_{2}\left(k_{x}\left(t_{i}\right) \Delta, k_{y}\left(t_{i}\right) \Delta\right) . \\
& {\left[\sum_{j=1}^{N} \mathrm{e}^{-\imath 2 \pi\left(k_{x}\left(t_{i}\right) x_{j}+k_{y}\left(t_{i}\right) y_{j}\right)}\left(c_{l j} f_{j}\right)\right] . }
\end{aligned}
$$

We can evaluate this forward model or its adjoint using $L$ NUFFT calls. This will be useful if the approximation (8) can be sufficiently accurate for a modest value of $L$. An intriguing option is the histogram PCA approach of [2].

\subsection{Iteration}

Combining either of the approximate signal models (7) or (9) with (2) yields the matrix-vector form

$$
\boldsymbol{y}=\boldsymbol{A x}+\boldsymbol{\varepsilon},
$$

just with somewhat different forms for the elements of the system matrix $\boldsymbol{A}$. Based on (5), the exact elements of $\boldsymbol{A}$ are given by

$$
\begin{aligned}
a_{i j}= & \operatorname{sinc}_{2}\left(k_{x}\left(t_{i}\right) \Delta, k_{y}\left(t_{i}\right) \Delta\right) . \\
& H\left(t_{i} g_{j}\right) \mathrm{e}^{-\imath \omega_{j} t_{i}} \mathrm{e}^{-\imath 2 \pi\left(k_{x}\left(t_{i}\right) x_{j}+k_{y}\left(t_{i}\right) y_{j}\right)} .
\end{aligned}
$$

We estimate $\boldsymbol{x}$ by minimizing a regularized least-squares cost function as follows:

$$
\begin{gathered}
\hat{\boldsymbol{x}}=\underset{\boldsymbol{x}}{\arg \min } \Psi(\boldsymbol{x}) \\
\Psi(\boldsymbol{x})=\frac{1}{2}\|\boldsymbol{y}-\boldsymbol{A} \boldsymbol{x}\|^{2}+\mathrm{R}(\boldsymbol{x}),
\end{gathered}
$$

where $\mathrm{R}(\boldsymbol{x})$ is a regularizing roughness penalty function that controls noise. The conjugate-gradient (CG) algorithm is an attractive approach for minimizing $\Psi$. Each iteration requires evaluating matrix-vector multiplications with $\boldsymbol{A}$ and $\boldsymbol{A}^{\prime}$. We perform this operations using the approximations (7) or (9) in conjunction with NUFFT operations [9].

\section{RESULTS}

To evaluate the proposed methods, we acquired human brain MR field maps as described in [8]. The scans were 64 by 64 by 40 slices, with $24 \mathrm{~cm}$ transaxial FOV and $4 \mathrm{~cm}$ axial FOV. (Thus the slice spacing was only $1 \mathrm{~mm}$.)

Fig. 1 and Fig. 2 show 12 of the the magnitude images and the corresponding field map images. Those field map images were formed using the regularized method described in [7]. Using central differences, we estimated the field map gradients, as shown in Fig. 3.

Fig. 4 shows the 2D histogram of $\left(\omega_{j}, g_{j}\right)$ values within the brain regions (those exceeding $1 \%$ of the maximum magnitude value). We design the approximation (8) using this histogram.

We will compare images reconstructed without throughplane gradient correction with images reconstructed using the exact model (5), which will be very slow. We will then explore the CT algorithm based on each of the two approximations (7) and (9) for various values of $L$.

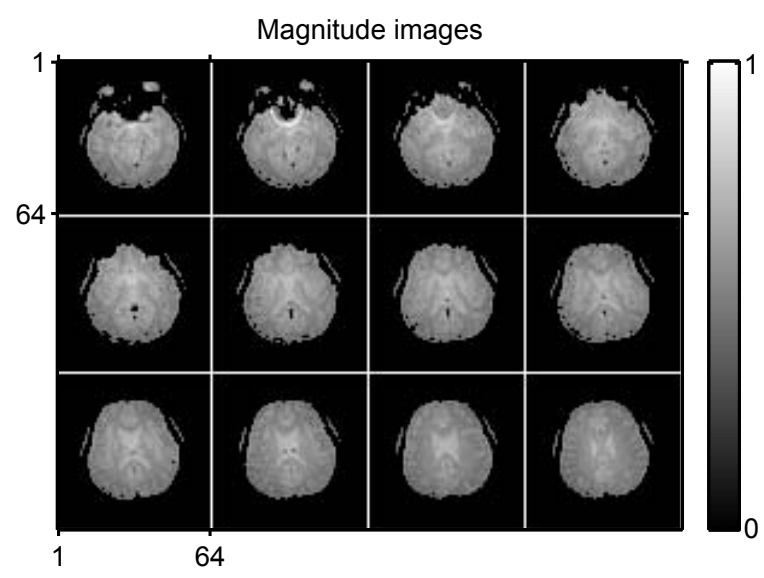

Fig. 1. Magnitude images used in simulation.

\section{DISCUSSION}

We have described a method for MR image reconstruction with compensation for field inhomogeneity and through-plane gradients of the field map.

The Fourier transform $H(\cdot)$ of the slice profile is simply the RF pulse shape (for small tip excitations), and we can choose from a variety of shapes. This will be an interesting avenue for future investigations. 


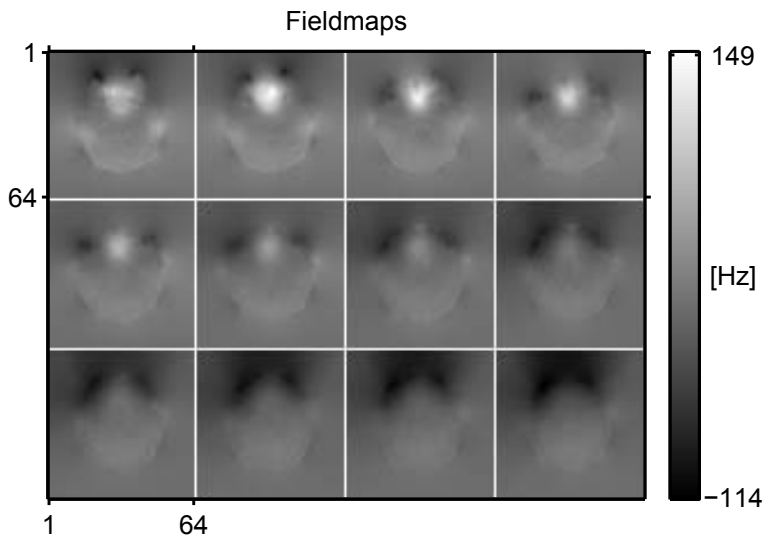

Fig. 2. Field maps used in simulation.

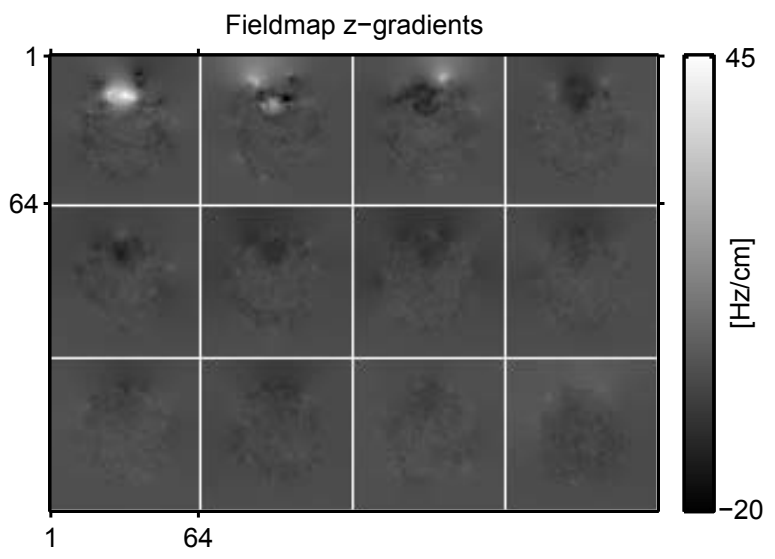

Fig. 3. Field map $z$-gradients.

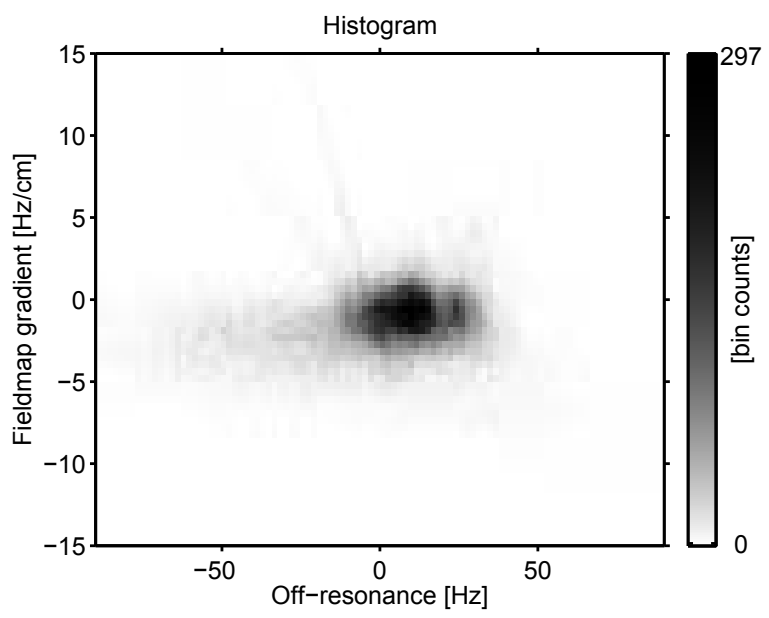

Fig. 4. Joint histogram of field map and its gradient.
A reviewer raised the question of whether field inhomogeneity gradients could affect the slice profile, akin to the "potato chip" effect [10]. This is an interesting question requiring further investigation. If there is a unique axial location $z_{0}(x, y)$ that is on-resonance for each transaxial coordinate $(x, y)$, then it should be possible to replace $z_{0}$ in (1) with this $z_{0}(x, y)$, and the remainder of the algorithm derivation should generalize relatively easily, leading to a reconstruction of the 2D function $f\left(x, y, z_{0}(x, y)\right)$ corresponding to a curved slice.

The linear expansion (4) would need modified if quadratic phase profiles are used to reduce susceptibility effects [11].

\section{REFERENCES}

[1] B. P. Sutton, D. C. Noll, and J. A. Fessler, "Fast, iterative image reconstruction for MRI in the presence of field inhomogeneities," IEEE Trans. Med. Imag., vol. 22, no. 2, pp. 178-88, Feb. 2003.

[2] J. A. Fessler, S. Lee, V. T. Olafsson, H. R. Shi, and D. C. Noll, "Toeplitz-based iterative image reconstruction for MRI with correction for magnetic field inhomogeneity," IEEE Trans. Sig. Proc., vol. 53, no. 9, pp. 3393-402, Sept. 2005.

[3] K. Sekihara, S. Matsui, and H. Kohno, "NMR imaging for magnets with large nonuniformities," IEEE Trans. Med. Imag., vol. 4, no. 4, pp. 193-9, Dec. 1985.

[4] D. C. Noll, J. A. Fessler, and B. P. Sutton, "Conjugate phase MRI reconstruction with spatially variant sample density correction," IEEE Trans. Med. Imag., vol. 24, no. 3, pp. 325-36, Mar. 2005.

[5] B. P. Sutton, Physics-based reconstruction of magnetic resonance images, Ph.D. thesis, Univ. of Michigan, Ann Arbor, MI, 48109-2122, Ann Arbor, MI, 2003.

[6] B. P. Sutton, D. C. Noll, and J. A. Fessler, "Compensating for within-voxel susceptibility gradients in BOLD fMRI," in Proc. Intl. Soc. Mag. Res. Med., 2004, p. 349.

[7] J. A. Fessler, D. Yeo, and D. C. Noll, "Regularized fieldmap estimation in MRI," in Proc. IEEE Intl. Symp. Biomed. Imag., 2006, pp. 706-9.

[8] C. Yip, J. A. Fessler, and D. C. Noll, "Advanced threedimensional tailored RF pulse for signal recovery in $T_{2}^{*}$ weighted functional magnetic resonance imaging," Mag. Res. Med., vol. 56, no. 5, pp. 1050-9, Nov. 2006.

[9] J. A. Fessler and B. P. Sutton, "Nonuniform fast Fourier transforms using min-max interpolation," IEEE Trans. Sig. Proc., vol. 51, no. 2, pp. 560-74, Feb. 2003.

[10] T. S. Sumanaweera, G. H. Glover, T. O. Binford, and J. R. Adler, "MR susceptibility misregistration correction," IEEE Trans. Med. Imag., vol. 12, no. 2, pp. 251-9, June 1993.

[11] Z. H. Cho and Y. M. Ro, "Reduction of susceptibility artifact in gradient-echo imaging," Mag. Res. Med., vol. 23, no. 1, pp. 193-200, Jan. 1992. 